\title{
An Integrated Framework for The Value Focused Thinking Methodology
}

\author{
Kweku-Muata Osei-Bryson \\ Department of Information Systems \\ Virginia Commonwealth University, U.S.A \\ KMOsei@,VCU.Edu
}

\begin{abstract}
In this paper we presented an integrated framework for the Value Focused Thinking (VFT) methodology that attempts to address issues that have not been adequately addressed. This framework provides several benefits including: the elicitation and high quality definition of objectives that incorporates organizational-oriented \& domainoriented concerns and knowledge, and the automatic generation of the alternate solutions that best satisfy the objectives, constraints and preference values. The proposed framework could contribute to a more effective application of the VFT methodology.
\end{abstract}

\section{INTRODUCTION}

The Value-Focused Thinking (VFT) methodology (Keeney [1] [2]), provides guidance on the formulation of objectives, an indispensable task in any decision making situation. VFT has been applied across a wide variety of domains such as project management (Barclay \& Osei-Bryson [3]), tourism management (Kajanus et al. [4] ),systems engineering (Boylan et al., [ 5]), ERP Systems (May,Dhillon \& Caldeira [6]), IS Security (Maitland, Barclay \& OseiBryson [7], Dhillon \& Torkzadeh [8]). Within the context of the VFT methodology (e.g. Barclay [9]) objectives are classified as being either a fundamental objective (FO) or a means-objective (MO), where each $\mathrm{MO}$ is an objective that is required in order to directly achieve its parent FO or another MO.

VFT can done in a top-down or bottom manner, with our focus in this paper being on the former. In a topdown approach Means Objectives (MO) are obtained from fundamental objectives (FO), by determining for each FO all the immediate lower level things that must be done satisfactorily (i.e. MO) in order to achieve the given FO. Lower level MOs can be obtained for next higher level MOs in a similar manner. The result is a network of objectives with the FOs at the root level and a subset of the MOs at the leaf level. Each leaf level MO can be considered to be equivalent to an actionable goal.

1. Frame the Decision Situation

a. Define the Decision Context: This is framed by the associated Administrative, Political \& Social structures

b. Identify the Objectives

c. Structure the Objectives into a MeansEnds Network

d. Specify Attributes

2. Preference Elicitation

3. Create Alternatives

4. Recommended Decision

5. Sensitivity Analysis

In this paper we present a new integrated framework for the VFT process that will address the following issues:

\section{$>$ Decision Context:}

Studies involving the application of the VFT methodology could be considered to fall into categories: a) those that attempt to identify Fundamental Objectives (FOs) \& Means Objectives (MOs) relevant to a given domain within specific situation organization (e.g. Barclay \& Osei-Bryson [3]); and b) those that those that attempt to identify FOs \& MOs that are generally relevant to a given domain (e.g. Dhillon \& Torkzadeh [8]). A fundamental concern with the latter approach is that VFT is to be applied within a particular decision context that is determined by relevant administrative, social, cultural \& political structures, and decision styles, and as such the decision contexts for a given domain (e.g. security) could vary across organizations.

$>$ Types of Relationships between Objectives: There are several types of relationships that could exist between objectives including:

- Parent-Child (PC) 
- Intrinsic Conflict (IC): The objectives conflict by their very nature (i.e. the relevant desired directions of the given pair of objectives cannot be simultaneously achieved, not because they compete for the same resources but because they instrinsically conflicting. An example of this is the intrinsic between the Confidentiality \& Availability objectives of a security plan: having maximum Confidentiality results in minimum Availability, and vice versa.

- Resource Conflict (RC): The given pair of Objectives utilize \& thus compete for one or more resources, and because of this fact the relevant desired directions of the given pair of objectives cannot be simultaneously achieved.

The traditional VFT process explicitly focuses on $\boldsymbol{P C}$ relationship types only, although both $\boldsymbol{I C} \&$ $\boldsymbol{R} \boldsymbol{C}$ are relevant to the Create Alternatives step of the VFT methodology. The approach presented in this paper will focus on all relationship types.

$>$ Quality of the Description of the Objectives: It is important that the objectives have important quality properties including Relevance, Completeness (i.e. for a given non-leaf objective, all of its relevant child Means Objectives must be specified), Non-Redundancy (i.e. No two objectives in the same tier should overlap), Specificity (i.e. must lead to an observable action, behavior or achievement)

The traditional VFT process does not explicitly focus on assessing all relevant quality dimensions.

$>$ Need to Create Values-based Alternatives:

Keeney [2] noted that "The first alternatives that come to mind are the obvious ones ... Truly different alternatives remain hidden in another part of the mind, unreachable by mere tweaking ... Focusing on the values that should be guiding the decision situation removes the anchor on the narrowly defined alternatives ... the means objectives are also meaningful ground to stimulate thinking about the objectives". We adopt these insights to design a method for the automatic generation of the alternatives that factors both the relevant preference values and constraints.

\section{OVERVIEW ON SOME SUPPORTING FRAMEWORKS}

In this section we present overviews on some of the supporting frameworks that could be utilized.

\subsection{The S.M.A.R.T Framework:}

Several frameworks have been proposed for evaluating the quality of a business objective including the SMART framework (Doran [10]) which suggested the following set of criteria:

- Specific: It must lead to an observable action, behavior or achievement that can be measured

- Measurable: Clearly defined metrics should be available for measuring the achievement of the objective. This is particularly relevant for the MOs

- Achievable: It must be achievable within the constraints of the available resources, knowledge \& time.

- Relevant: Must be relevant to the broader goals of the organization

○ Time-bound: there should be specific deadlines for the achievement of the objective. This is particularly relevant for the MOs.

A review of previous VFT papers shows that often the MOs are not expressed in a manner that can be considered to be Time-bound. Further the Achievability criteria is often not considered particularly with respect to the Intrinsic Conflict (IC) and Resource Conflict (RC) types of constraints.

\subsection{Some Relevant Organizational Issues}

The reader may recall that the Decision Context is framed by the associated Administrative, Political \& Social structures. Thus there are several types of organizational issues that have to be accommodated in the definition of the objectives. We will focus on a few of these below.

\subsubsection{Overview on the Organizational Types:} Courtney [11] presented a set of organizational types, and corresponding organizational decision-making style. It seems reasonable to expect that the organizational decision-making style would impact on the feasibility and definition of the MOs. 


\begin{tabular}{|c|c|c|c|c|c|}
\hline & Leibniz & Locke & Kant & Hegel & Singer \\
\hline $\begin{array}{l}\text { Organizational } \\
\text { Decision-Making } \\
\text { Style }\end{array}$ & $\begin{array}{ll}\text { - } & \text { Formal } \\
\text { - } & \text { Analytical } \\
\text { Bureaucratic }\end{array}$ & $\begin{array}{ll}\text { - } & \text { Open } \\
\text { - } & \text { Communicative } \\
\text { - } & \text { Consensual }\end{array}$ & $\begin{array}{ll}\text { - } & \text { Open } \\
\text { - } & \text { Analytical }\end{array}$ & - Conflictual & $\begin{array}{ll}\text { - } & \text { Teleological } \\
\text { - } & \text { Cooperative } \\
\text { - } & \text { Ethical }\end{array}$ \\
\hline
\end{tabular}

\subsubsection{Overview on Individual Decision Styles:}

Rowe \& Boulgarides [12] identified four major categories of individual decision styles. Martinsons \& Davison [13] observed that in different cultures, different individual decision styles are dominant. It seems reasonable to expect that in some settings the individual decision-making style would impact on the feasibility and definition of the MOs.

\begin{tabular}{|l|l|}
\hline Style & Description \\
\hline Analytical & $\begin{array}{l}\text { Achievement oriented without the need for external rewards; make decisions slowly because } \\
\text { orientation to examine the situation thoroughly and consider many alternatives systematically }\end{array}$ \\
\hline Behavioral & $\begin{array}{l}\text { Strong people orientation, driven primarily by a need for affiliation; typically receptive to suggestions, } \\
\text { willing to compromise, and prefer loose controls }\end{array}$ \\
\hline Conceptual & $\begin{array}{l}\text { Achievement \& people oriented with the need for external rewards; make decisions slowly because } \\
\text { orientation to examine the situation thoroughly and consider many alternatives systematically }\end{array}$ \\
\hline Directive & Results and power oriented but prefer to consider a limited number of alternatives that they consider \\
\hline
\end{tabular}

\subsubsection{Overview on the Cultural Dimensions:}

Hofstede [14] defined a set of cultural dimensions that could impact the behaviors of organizational actors that are outlined below. The characteristics of a given national culture may mean that some Means Objectives are infeasible in that context. It is therefore important that cultural issues be taken into consideration.

\begin{tabular}{|l|l|}
\hline Dimension & Description \\
\hline Power Distance & Reflects the extent to which the members in a society accept the unequal distribution of power \\
\hline $\begin{array}{l}\text { Individualism- } \\
\text { Collectivism }\end{array}$ & $\begin{array}{l}\text { Reflects the degree to which people are able and prefer to achieve an identity and status on their } \\
\text { own rather than through group memberships }\end{array}$ \\
\hline $\begin{array}{l}\text { Masculinity- } \\
\text { Femininity }\end{array}$ & $\begin{array}{l}\text { Reflects the degree to which assertiveness and achievement are valued over nurturing and } \\
\text { affiliation }\end{array}$ \\
\hline $\begin{array}{l}\text { Uncertainty } \\
\text { Avoidance }\end{array}$ & Reflects discomfort with ambiguity and incomplete information \\
\hline
\end{tabular}

\subsubsection{Overview on Organizational Perspectives:}

Kaplan \& Norton ([15] [16]) presented the Balanced Scorecard (BSC) Model that involves 4 perspectives presented in the table below. An exploration of these perspectives could lead to the discovery of important organizational values and objectives.

\begin{tabular}{|l|l|}
\hline Perspective & Description \\
\hline Customer & How do the customers see the organisation? \\
\hline Internal Business & What must the organisation excel at? \\
\hline Financial & How does the organisation look to the shareholders? \\
\hline Innovation \& Learning & How can the organisation continue to improve and create value? \\
\hline
\end{tabular}




\subsection{Probing Questions}

The importance of 'probing' questions in the elicitation process has been recognized by previous VFT researchers. For example Step 1 of the research approach of Dhillon \& Torkzadeh [8] involves using 'probes to develop in depth understanding' of the decision problem. In this subsection we list some relevant probing questions; these questions are influenced by the material presented in subsections $2.1 \& 2.2$.

1. Frame the Decision Situation

a. Define the Decision Context: This is framed by the associated Administrative, Political \& Social structures

i. What is the organization type of your organization in the sense of Courtney [11]?

ii. What is the nature of the problem \& its Environment?

- Who are the major relevant external players (i.e. Customers, Vendors, Competitors, Regulators)?

- What are the Economic, Technical, Time \& other resource factors that appear to be relevant?

iii. Who are the decision-makers (DMs) and what are their individual decision styles?

iv. What are the DM's values?

v. Which groups would be impacted by the decision(s)? Which groups would have to implement the decision(s)? Which groups (internal or external) could constrain decision options?

vi. What are some known decision alternatives?

b. Identify the Objectives: This requires that in each case an Object is identified as well as the Direction of Preference. Questions that could guide the identification include:

i. What are the ultimate objectives?

ii. What are the perceived Best Practices for the given decision problem domain? What are the previously identified Objectives for the given decision problem domain?

iii. What are some concerns from a Financial perspective?

iv. What are some concerns from an External Stakeholder perspective?

- WHO are your Customers \& WHAT would they be concerned about? What would your Vendors be concerned about?

- What would your Competitors be concerned about? What would your Regulators be concerned about?

- What would your Shareholders be concerned about?

v. What are some concerns from an Internal Stakeholder perspective?

- What are some concerns from a Cultural perspective?

- What are some concerns from a Decision Style perspective?

- What are some concerns from a Ethical perspective?

- What are some concerns from a Health \& Safety perspective?

vi. What are some concerns from a Learning \& Innovation perspective?

vii. What are some concerns from a Scheduling perspective?

viii. What are some concerns from a Legal perspective?

ix. What are some concerns from a Technical/Technological perspective?

\section{DESCRIPTION OF THE INTEGRATED EXTENDED VFT METHODOLOGY}

Below we present a description of the proposed integrated framework for the VFT methodology. The reader should note that the first two phases (i.e. BU \& DU) present probes that could be used to develop an in-depth understanding of the decision problem. 


\section{1 Business Understanding (BU):}

This phase is concerned with exposing \& recording the organizational factors that should be included in the framing of the Decision Context. Relevant probing questions include:

- What are the ultimate objectives for the given decision problem domain? What are the previously identified Objectives for the given decision problem domain?

- What are the significant concerns from a Financial perspective?

- What are the significant concerns from an External Stakeholder perspective?

- What are the significant concerns from an Internal Stakeholder perspective?

- What are the significant concerns from a Learning \& Innovation perspective?

- What are the significant concerns from a Scheduling perspective?

- What are the significant concerns from a Legal perspective?

Steps in this phase would include:

1. Obtain \& Review Organization Mission \& Vision statements, Organization Chart / Organizational Ontology, Main Products/Services

2. Identify relevant Internal \& External Stakeholders

3. Determine the Main Decision Styles of relevant Internal Stakeholders

4. Use the relevant prompting questions to identify the concerns from the 6 organizational perspectives listed above. Record these concerns

\subsection{Domain Understanding (DU):}

This phase is concerned with exposing \& recording the domain issues that should be included in the framing of the Decision Context. Relevant probing questions include:

- What are the perceived Concepts for the domain of the given decision problem?

- What are the previously identified Objectives for the given decision problem domain?

- What are the perceived Best Practices for the given decision problem domain?

- What are some concerns from a Learning \& Innovation perspective?
- What are some concerns from a Legal perspective?

- What are some concerns from a Technical/Technological perspective?

Steps in this phase would include:

1. Review relevant domain knowledge bases.

2. Use the relevant prompting questions to identify relevant domain-oriented Concepts, Best Practices, Fundamental \& Means Objectives, and concerns from the 4 perspectives listed above. Record this information.

\subsection{Modeling Objectives (MD):}

This phase has 3 sub-phases as described below.

\subsubsection{Initial Identification of Objectives}

1. Use the recorded information that resulted from the Business Understanding \& Domain Understanding phases to identify Objectives that meet the Relevance criteria.

2. Refine definition of each Objective so that it satisfies the Specificity property.

\subsubsection{Classification \& Refinement of Objectives}

1. Classify each Objective in the current set of Objectives as being a $F O$ or a $M O$, and identify the associated set of Parent-Child $(P C)$ relationships.

2. For each $F O$, determine if its current set of supporting child $M O$ s is sufficient for the given $F O$ to satisfy the Completeness property. If the Completeness property is not satisfied for a given $F O$ then identify the remaining supporting child $M O$ s so that this property is satisfied. Update the associated set of Parent-Child $(P C)$ relationships.

3. For each $M O$ that is a parent of other $M O \mathrm{~s}$ determine if its current set of supporting child $M O$ s is sufficient for the given $M O$ to satisfy the Completeness property. If the Completeness property is not satisfied for a given $F O$ then identify remaining supporting child $M O$ s so that this property is satisfied. Update the associated set of Parent-Child $(P C)$ relationships.

4. For each $M O$ use the Why-Is-It-Important (WITI) test to determine if it has any the other objective (i.e. another $M O$ or a $F O$ ) is also its parent. Update the set of Parent-Child $(P C)$ relationships.

5. Review the current set of $M O \mathrm{~s}$ in order to identify the leaf-level $M O$ s.

6. For each leaf-level $M O$, refine its definition so that it satisfies the Measurability, Achievability and Time-boundedness properties. 
It should be note that after the completion of this subphase that the Completeness property and the 5 SMART properties would have been satisfied.

\subsubsection{Identification of Achievement Processes (APs)}

1. Define an ordered discrete set of qualitative performance levels will be specified (e.g. High, Medium, Low).

2. For each leaf-level $M O$ :

a. Use the Goal Question Metric (e.g. Basili, Caldiera, \& Rombach [17]) method to identify relevant performance measures (i.e. attributes);

b. For each of its corresponding qualitative performance level $\ell$, identify the combinations of attribute levels that are associated with level $\ell$. Let $\mathrm{H}_{\mathrm{j} \ell}$ be the corresponding set of attribute level combinations;

c. Identify a set of Achievement Processes that could be used realize the various performance levels of the given leaflevel $M O$.

d. Estimate the cost and requirements of depletable resource necessary for a given Achievement Process to realize each performance level of the MO.

e. Identify any additional constraints (e.g. Legal, Technological, Scheduling) based on the Concerns/Issues identified in BU \& DU phases that relate to the achievement of relevant performance levels.

It should be noted that while a $M O$ describes $W H A T$ is desired, a corresponding Achievement Process (AP) would describe $H O W$ the given $W H A T$ could be achieved. Description of an $A P$ includes its method as well as a description of the resources that are required to achieve the relevant performance levels of the $M O$. It should be noted that resource requirements that are estimated in this sub-phase could be used for the identification of Resource Conflict $(R C)$ relationships. Further the fact that at this stage each FO \& MO satisfies the Specificity property then relevant information is also available to identify any Intrinsic Conflict $(I C)$ relationship between performance levels of pairs of Objectives.

\subsection{Elicit Preference Information}

1. Use a pairwise comparisons approach such as that used in the AHP to determine, $\mathrm{w}_{\mathrm{i}}$, the relative importance of each FO “i'.

2. For each FO i, use a pairwise comparisons approach to determine the relative value $\mathrm{v}_{\mathrm{ik}}$ of each possible score level $\mathrm{k}$.

\subsection{Generate \& Evaluate Alternatives:}

This phase has two sub-phases. The first sub-phase focuses on the formulation of a mathematical programming problem (MPP) that would be used for generating the alternatives that are reflective of the preference values and also relevant constraints. This MPP could also be use to do What-If and sensitivity analyses. The second sub-phase outlines the procedure for formulating \& solving the MPP to generate and evaluate alternate solutions, including 'near optimal' ones

\subsubsection{Mathematical Programming Formulation}

$\mathrm{I}$ is the set of Objectives; $\mathrm{I}_{\mathrm{FO}}$ is the subset of Fundamental Objectives (FO); $\mathrm{I}_{\mathrm{MO}}$ is the subset of Means Objectives (MO); I = $\mathrm{I}_{\mathrm{FO}} \cup \mathrm{I}_{\mathrm{MO}} ; \mathrm{I}_{\mathrm{FO}} \cap$ $\mathrm{I}_{\mathrm{MO}}=\varnothing$.

$>\mathrm{V}_{\mathrm{ik}}$ is the value associated with FO "i" being achieved at level $\mathrm{k} \in \mathrm{K}_{\mathrm{i}}$.

$>\mathrm{x}_{\mathrm{ik}}$ is a binary variable such that $\mathrm{x}_{\mathrm{ik}}=1$ indicates that Objective " $i$ " has been achieved at level " $k$ "; and $\mathrm{x}_{\mathrm{ik}}=0$ otherwise.

\section{Parent-Child Constraints on Achievement of Performance Levels:}

$>\mathrm{M}_{\mathrm{ik}}$ is the set of combinations of MOs each at a specified performance level $\ell$, such that each combination in $\mathrm{M}_{\mathrm{ik}}$ would result Objective i being achieved at performance level $\mathrm{k}$.

- For each $m \in M_{i k}, J_{i k m}$ is a set of MOs, each a child of Objective $i$ and each at a performance level that taken together would result in Objective $i$ being achieved at level $\mathrm{k}$.

○ $\quad \mathrm{Z}_{\mathrm{ikm}}$ is a binary variable such that $\mathrm{Z}_{\mathrm{ikm}}=1$ indicates that each MO " $\mathrm{j}$ " in $\mathrm{J}_{\mathrm{ikm}}$ is at the relevant performance level $\ell$; and $\mathrm{z}_{\mathrm{ikm}}=0$ otherwise. 
1a: $\mathrm{z}_{\mathrm{ikm}}-\mathrm{x}_{\mathrm{j} \ell} \leq 0 \quad \forall \mathrm{m} \in \mathrm{M}_{\mathrm{ik}},(\mathrm{j}, \ell) \in \mathrm{J}_{\mathrm{ikm}}$ 1b: $\Sigma_{(\mathrm{j}, \ell)=\mathrm{Jikm}} \mathrm{X}_{\mathrm{j} \ell}-\mathrm{Z}_{\mathrm{ikm}} \leq\left(\left|\mathrm{J}_{\mathrm{ikm}}\right|-1\right) \quad \forall \mathrm{m} \in \mathrm{M}_{\mathrm{ik}}$

Objective " $\mathrm{i}$ " is achieved at level " $\mathrm{k}$ " only if at least one combination in $\mathrm{M}_{\mathrm{ik}}$ is realized:

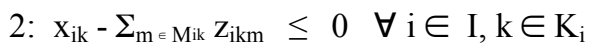

$>$ Each objective "i" achieves exactly one of its allowable levels $\mathrm{k} \in \mathrm{K}_{\mathrm{i}}$

3: $\sum_{\mathrm{k} \in \mathrm{Ki}} \mathrm{x}_{\mathrm{ik}} \quad=1 \quad \forall \quad \mathrm{i} \in \mathrm{I}$

Resource Conflicts:

$\mathrm{q}_{\mathrm{rj} \ell}$ is the minimum amount of depletable resource " $r$ " that is required in order for MO " $j$ " to be achieved at level " $\ell$ ", and $\mathrm{q}_{\mathrm{r}}$. be the total available amount of resource "r":

(4) $\sum_{\mathrm{j} \text { IMO }} \sum_{\ell=\mathrm{Ki}} \mathrm{q}_{\mathrm{rj} \ell \mathrm{X}_{\mathrm{j} \ell}} \quad \leq \mathrm{q}_{\mathrm{r} .} \forall \quad \forall \quad \mathrm{r} \in \mathrm{R}$

\section{Intrinsic Conflicts:}

$>\quad \mathrm{P}$ is the set of pairs of Objectives, $\left(\mathrm{i}_{1}, \mathrm{i}_{2}\right)$ that have Intrinsic Conflicts where $i_{1} \in I \&$ $\mathrm{i}_{2} \in \mathrm{I}$ such that if $\mathrm{i}_{1} \in \mathrm{I}$ achieves level $\mathrm{k}_{1}$ then $\mathrm{i}_{2} \in \mathrm{I}$ cannot achieve level $\mathrm{k}_{2}$.

(5) $\mathrm{x}_{\mathrm{i} 1, \mathrm{k} 1}+\mathrm{x}_{\mathrm{i} 2, \mathrm{k} 2} \leq 1 \quad \forall\left(\mathrm{i}_{1}, \mathrm{k}_{1}, \mathrm{i}_{2}, \mathrm{k}_{2}\right) \in \mathrm{P}$

\section{Integer Programming Problem (MPP) to} Generate Alternatives:

$\mathrm{IP}_{\text {GenAlt }}: \operatorname{Max}\left\{\Sigma_{\mathrm{i}=\mathrm{IFO}} \Sigma_{\mathrm{k} \in \mathrm{Ki}} \mathrm{W}_{\mathrm{i}} \mathrm{v}_{\mathrm{ik}} \mathrm{X}_{\mathrm{ik}} \mid(1)-(5), \&\right.$ binary restriction on all variables $\}$

\subsubsection{Procedure for Generating Alternatives}

1. Formulate \& Solve problem $P_{\text {GenAlt }}$.

2. Given the initial optimal solution to problem $\mathrm{P}_{\text {GenAlt, }}$ generate other alternate optimal solutions if they exist.

3. Use What-If and/or Sensitivity Analysis to generate other alternate though near optimal solutions to problem $\mathrm{P}_{\text {GenAlt }}$.

\section{ILLUSTRATIVE EXAMPLE}

In this section we present an illustrative example that applies the procedure for generating alternatives that satisfy the three types of constraints. Figure 1 displays the Means-Ends Objective Network (a hierarchy in this case) for an information systems security.

For each Objective, exactly 1 Performance Level can be achieved. Further there are three possible performance levels (i.e. High (3), Medium (2), and
Low (1)) for each Objective. Table 1 displays the constraints that represent these facts. Rather than use " $x$ " and " $y$ " for the variable names we have the variable that represents each Means Objective (MO) level begin with " $\mathrm{M}$ ", and the variable that represents each Fundamental Objective (FO) level begin with "F" where each variable is a $0 / 1$ integer variable.

Figure 1: Means-Ends Objectives Network

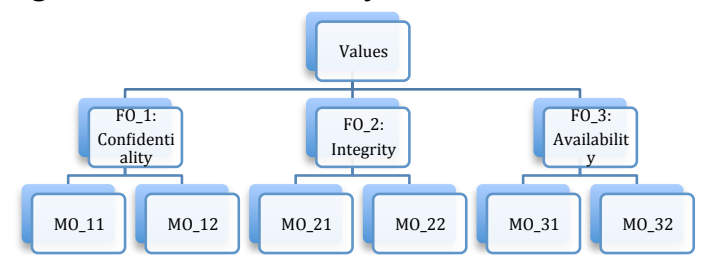

Table 1: Select One Performance Level Constraint

\begin{tabular}{|c|c|c|}
\hline \multirow[t]{6}{*}{ Means } & MO_11 & $\mathrm{M} 111+\mathrm{M} 112+\mathrm{M} 113=1$ \\
\hline & MO_12 & $\mathrm{M} 121+\mathrm{M} 122+\mathrm{M} 123=1$ \\
\hline & MO 21 & $\mathrm{M} 211+\mathrm{M} 212+\mathrm{M} 213=1$ \\
\hline & MO 22 & $\mathrm{M} 221+\mathrm{M} 222+\mathrm{M} 223=1$ \\
\hline & MO_31 & $\mathrm{M} 311+\mathrm{M} 312+\mathrm{M} 313=1$ \\
\hline & MO 32 & $\mathrm{M} 321+\mathrm{M} 322+\mathrm{M} 323=1$ \\
\hline \multirow{3}{*}{$\begin{array}{l}\text { Fundament } \\
\text { al }\end{array}$} & FO_1 & $\mathrm{F} 11+\mathrm{F} 12+\mathrm{F} 13=1$ \\
\hline & FO_2 & $\mathrm{F} 21+\mathrm{F} 22+\mathrm{F} 23=1$ \\
\hline & FO_3 & $\mathrm{F} 31+\mathrm{F} 32+\mathrm{F} 33=1$ \\
\hline
\end{tabular}

Tables $2-4 \mathrm{~b}$ displays the various other types of constraints: Parent-Child Constraints on Achievement of Performance Levels (Table2), Intrinsic Conflict (Table3), and a financial Resource Conflict (Tables 4a \& 4b). Table 5a displays the Weight for each Fundamental Objective (FO) and the Value associated with achieving each performance level of each FO. The value of the highest level (i.e.. Level 3) of each Fundamental Objective is set to 100 , with the value of its lower levels be relative to the value of the highest level. Methods such as the Analytic Hierarchy Process (e.g. Saaty [18], Bryson [19] could be used to both derive the relative weight of each FO as well for each FO the relative value of its lower performance levels with respect to its highest performance level. In the Table $5 \mathrm{~b}$, the coefficient of each variable is its weighted Value. 
Table 2: Parent-Child Constraints

\begin{tabular}{|c|c|}
\hline $\begin{array}{l}\text { If } \mathrm{MO}_{-} 11 \& \mathrm{MO}_{-} 12 \\
\text { are both at Level } 3 \text { then } \\
\text { FO_1 is at Level } 3 ;\end{array}$ & $\begin{array}{l}\mathrm{M} 113+\mathrm{M} 123-\mathrm{F} 13<=1 \\
\mathrm{~F} 13-\mathrm{M} 113<=0 \\
\mathrm{~F} 13-\mathrm{M} 123<=0\end{array}$ \\
\hline $\begin{array}{l}\text { If } \mathrm{MO}_{-} 11 \& \mathrm{MO}_{\overline{1}} 12 \\
\text { are both at Level } 1 \text { then } \\
\mathrm{FO} 1 \text { is at Level } 1 ;\end{array}$ & $\begin{array}{l}\text { M111+ M121 - F11<= } \\
\text { F11 - M111<=0 } \\
\text { F11-M121 }=0\end{array}$ \\
\hline $\begin{array}{l}\text { If } \mathrm{MO}_{-} 21 \text { \& } \mathrm{MO}_{2} 22 \\
\text { are both at Level } 3 \text { then } \\
\mathrm{FO}_{2} 2 \text { is at Level } 3 ;\end{array}$ & $\begin{array}{l}\mathrm{M} 213+\mathrm{M} 223-\mathrm{F} 23<=1 \\
\mathrm{~F} 23-\mathrm{M} 213<=0 \\
\mathrm{~F} 23-\mathrm{M} 223<=0\end{array}$ \\
\hline $\begin{array}{l}\text { If } \mathrm{MO} 21 \& \mathrm{MO}_{-} 22 \\
\text { are both at Level } 1 \text { then } \\
\mathrm{FO} 2 \text { is at Level } 1 ;\end{array}$ & $\begin{array}{l}\mathrm{M} 211+\mathrm{M} 221-\mathrm{F} 21<=1 \\
\mathrm{~F} 21-\mathrm{M} 211<=0 \\
\mathrm{~F} 21-\mathrm{M} 221<=0\end{array}$ \\
\hline $\begin{array}{l}\text { If } \mathrm{MO} 31 \& \mathrm{MO} 32 \\
\text { are both at Level } 3 \text { then } \\
\text { FO_3 is at Level } 3 ;\end{array}$ & $\begin{array}{l}\mathrm{M} 313+\mathrm{M} 323-\mathrm{F} 33<=1 \\
\mathrm{~F} 33-\mathrm{M} 313<=0 \\
\mathrm{~F} 33-\mathrm{M} 323<=0\end{array}$ \\
\hline $\begin{array}{l}\text { If } \mathrm{MO} 31 \text { \& } \mathrm{MO}_{-} 32 \\
\text { are both at Level } 1 \text { then } \\
\mathrm{FO} 3 \text { is at Level } 1 ;\end{array}$ & $\begin{array}{l}\text { M311 } \mathrm{M} 321-\mathrm{F} 31<=1 \\
\text { F31-M311<=0 } \\
\text { F31-M321<=0 }\end{array}$ \\
\hline
\end{tabular}

Table 3: Intrinsic Conflict Constraint Confidentiality (FO_1) \& $\quad \mathrm{F} 13+\mathrm{F} 33<=1$ Availability (FO 3) cannot both be at Level 3

Table 4a: Objective Level Achievement Costs

\begin{tabular}{|c|c|c|c|}
\hline Fundamental & Means & Level & Cost \\
\hline \multirow{6}{*}{$\begin{array}{c}\text { Confidentiality } \\
\text { (C) }\end{array}$} & \multirow{3}{*}{$\mathrm{MO} \_11$} & 3 & 110 \\
\hline & & 2 & 80 \\
\hline & & 1 & 60 \\
\hline & \multirow[t]{3}{*}{ MO_12 } & 3 & 85 \\
\hline & & 2 & 75 \\
\hline & & 1 & 55 \\
\hline \multirow{6}{*}{$\begin{array}{l}\text { Integrity } \\
\text { (I) }\end{array}$} & \multirow[t]{3}{*}{ MO_21 } & 3 & 85 \\
\hline & & 2 & 75 \\
\hline & & 1 & 65 \\
\hline & \multirow[t]{3}{*}{ MO_22 } & 3 & 80 \\
\hline & & 2 & 70 \\
\hline & & 1 & 60 \\
\hline \multirow{6}{*}{$\begin{array}{c}\text { Availability } \\
\text { (A) }\end{array}$} & \multirow[t]{3}{*}{ MO_31 } & 3 & 105 \\
\hline & & 2 & 90 \\
\hline & & 1 & 65 \\
\hline & \multirow[t]{3}{*}{ MO_32 } & 3 & 110 \\
\hline & & 2 & 85 \\
\hline & & 1 & 70 \\
\hline
\end{tabular}

Table 4b: Financial Resource Constraint

110M113+80M112+60M111+
$85 \mathrm{M} 123+75 \mathrm{M} 122+55 \mathrm{M} 121+$
$85 \mathrm{M} 213+75 \mathrm{M} 212+65 \mathrm{M} 211+$
$80 \mathrm{M} 223+70 \mathrm{M} 222+60 \mathrm{M} 221+$
$105 \mathrm{M} 313+90 \mathrm{M} 312+65 \mathrm{M} 311+$
$110 \mathrm{M} 323+85 \mathrm{M} 322+70 \mathrm{M} 321$

Table 5a: Weights \& Achievement Level Values

\begin{tabular}{|l|c|c|c|}
\hline Fundamental Objective & Weight & Level & Value \\
\hline Confidentiality (C) & 0.37 & 3 & 100.00 \\
\cline { 3 - 4 } & & 2 & 85.00 \\
\cline { 3 - 4 } & & 1 & 65.00 \\
\hline Integrity (I) & \multirow{2}{*}{0.30} & 3 & 100.00 \\
\cline { 3 - 4 } & & 2 & 88.00 \\
\cline { 3 - 4 } & & 1 & 75.00 \\
\hline Availability (A) & \multirow{2}{*}{0.33} & 3 & 100.00 \\
\cline { 3 - 4 } & & 2 & 76.00 \\
\cline { 3 - 4 } & & 1 & 55.00 \\
\hline
\end{tabular}

Table 5b: Objective Function of IP Problem $37.00 \mathrm{~F} 13+31.45 \mathrm{~F} 12+24.05 \mathrm{~F} 11+30.00 \mathrm{~F} 23+$ $26.40 \mathrm{~F} 22+22.500 \mathrm{~F} 21+33.00 \mathrm{~F} 33+25.08 \mathrm{~F} 32+$ $18.15 \mathrm{~F} 31$

In Table 6 we display the results of solving the IP problem under 3 scenarios: None (i.e. no additional constraint), Confidentiality must be at its top performance level (i.e. Set FO_1 to Level 3), and Integrity must be at its top performance level (i.e. Set FO_2 to Level 3). For scenario, the performance levels of the $F O$ s and $M O$ s are provided. Since for each $\mathrm{MO}$ its corresponding Achievement Process would have previously identified then results generated by the Procedure for Generating Alternatives could be used to identify the performance levels of the relevant Achievement Process that corresponds to the given set of $M O$ s performance levels.

\section{CONCLUSION}

In this paper we presented an integrated framework for the Value Focused Thinking methodology that attempts to address significant issues that have not been adequately addressed. This framework provides several benefits including: the elicitation and high quality definition Objectives that accommodate organizational-oriented \& domain-oriented concerns 
and knowledge; and the automatic generation of the Alternate solutions that best satisfies the three types of relationship constraints and the preference values. It also offers the option of sensitivity and "What-If" analysis. This new VFT framework could contribute to a more effective application of the VFT methodology that is being increasingly used in a variety of situations (e.g. [3], [4], [5], [6], [7], [8], [20]).

Table 6: Description of the Generated Alternatives

\begin{tabular}{|c|c|c|c|c|c|}
\hline \multirow[t]{2}{*}{ Restriction } & \multirow[t]{2}{*}{ Value } & \multicolumn{2}{|c|}{ Fundamental } & \multicolumn{2}{|l|}{ Means } \\
\hline & & Obj & Lvl & Obj & Lvl \\
\hline \multirow[t]{6}{*}{ None } & \multirow[t]{6}{*}{90.85} & \multirow[t]{2}{*}{ FO_1 } & \multirow[t]{2}{*}{2} & MO_11 & 2 \\
\hline & & & & $\mathrm{MO} \_12$ & 1 \\
\hline & & \multirow[t]{2}{*}{$\mathrm{FO}_{2} 2$} & \multirow[t]{2}{*}{2} & MO 21 & 1 \\
\hline & & & & MO_22 & 3 \\
\hline & & \multirow[t]{2}{*}{$\mathrm{FO}_{-} 3$} & \multirow[t]{2}{*}{3} & MO_31 & 3 \\
\hline & & & & MO 32 & 3 \\
\hline \multirow{6}{*}{$\begin{array}{l}\text { Set FO_1 } \\
\text { to Level } 3\end{array}$} & \multirow[t]{6}{*}{88.48} & \multirow[t]{2}{*}{$\mathrm{FO}_{-1}$} & \multirow[t]{2}{*}{3} & MO_11 & 3 \\
\hline & & & & MO_12 & 3 \\
\hline & & \multirow[t]{2}{*}{$\mathrm{FO}_{-} 2$} & \multirow[t]{2}{*}{2} & MO 21 & 3 \\
\hline & & & & MO_22 & 1 \\
\hline & & \multirow[t]{2}{*}{ FO_3 } & \multirow[t]{2}{*}{2} & MO_31 & 2 \\
\hline & & & & MO_32 & 1 \\
\hline \multirow{6}{*}{$\begin{array}{l}\text { Set FO_2 } \\
\text { to Level } 3\end{array}$} & \multirow[t]{6}{*}{87.05} & \multirow[t]{2}{*}{$\mathrm{FO}_{-} 1$} & \multirow[t]{2}{*}{1} & MO_11 & 1 \\
\hline & & & & MO_12 & 1 \\
\hline & & \multirow[t]{2}{*}{$\mathrm{FO}_{2} 2$} & \multirow[t]{2}{*}{3} & MO_21 & 3 \\
\hline & & & & MO_22 & 3 \\
\hline & & \multirow[t]{2}{*}{$\mathrm{FO}_{3} 3$} & \multirow[t]{2}{*}{3} & MO_31 & 3 \\
\hline & & & & MO 32 & 3 \\
\hline
\end{tabular}

\section{REFERENCES}

1. Keeney, R. L. (1992). Value-Focused Thinking: $\boldsymbol{A}$ Path to Creative Decision Making: Harvard University Press.

2. Keeney, R. L. (1996) "Value-Focused Thinking: Identifying Decision Opportunities and Creating Alternatives", European Journal of Operational Research 92, 537-549.

3. Barclay, C. \& Osei-Bryson, K.-M. (2010) "Project Performance Development Framework: An Approach for Developing Performance Criteria \& Measures for Information Systems (IS) Projects", International Journal of Production Economics 124:1, 272-292.

4. Kajanus, M., J. Kangas, \& Kurtilla, M. (2004) "The Use of Value Focused Thinking and the A'WOT Hybrid Method in Tourism Management", Tourism Management 25:4, 499-506

5. Boylan, G. L., E. S. Tollefson, Kwinn, M., \& Guckert, R. (2006) "Using Value-Focused Thinking to Select a Simulation Tool for the Acquisition of Infantry Soldier Systems",
Systems Engineering 9:3. 199 - 212

6. May, J., Dhillon, G., \& Caldeira, M. (2013). Defining value-based objectives for ERP systems planning. Decision Support Systems, 55(1), 98-109.

7. Barrett-Maitland, N., Barclay, C., \& Osei-Bryson, K. M. (2016). Security in Social Networking Services: A Value-Focused Thinking Exploration in Understanding Users' Privacy and Security Concerns. Information Technology for Development, 22(3), 464-486.

8. Dhillon, G. \& Torkzadeh, G. (2006) "Value-focused assessment of information system security in organizations", Information Systems Journal 16:3, 293-314.

9. Barclay, C. (2014) "Overview of the Value-focused Thinking methodology". In Advances in Research Methods for Information Systems Research (pp. 183196). Springer US.

10. Doran, G. (1981) "There's a S.M.A.R.T. Way to Write Management Goals and Objectives", Management Review 70:1, 35-36.

11. Courtney, J. (2001) "Decision making and knowledge management in inquiring organizations: toward a new decision-making paradigm for DSS", Decision Support Systems 31, 17-38.

12. Rowe, A. J., \& Boulgarides, J. D. (1983) "Decision Styles - A Perspective", Leadership \& Organization Development Journal, 4(4), 3-9.

13. Martinsons, M. \& Davison, R. (2007) "Strategic decision making and support systems: Comparing American, Japanese and Chinese management", Decision Support Systems 43, 284-300.

14. Hofstede, G. (1980) Culture's Consequences: International Differences in Work-Related Values, Sage Publications, Beverly Hills, CA.

15. Kaplan, R. \& Norton, D. (1992) "The Balanced Scorecard: Measures That Drive Performance", Harvard Business Review 70:1, 71-79.

16. Kaplan, R. S., \& Norton, D. P. (2001). The strategyfocused organization: How balanced scorecard companies thrive in the new business environment. Harvard Business Press.

17. Basili, V., Caldiera, G., \& Rombach, H (1994) "The Goal Question Metric Approach", Encyclopedia of Software Engineering.

18. Bryson, N. (1996) "Group Decision-Making and the Analytic Hierarchy Process: Exploring the ConsensusRelevant Information Content", Computers \& Operations Research 23, 27-35

19. Saaty, T. (1980) The Analytic Hierarchy Process: Planning, Priority Setting, Resource Allocation, McGraw-Hill, NY.

20. Osei-Bryson, K.-M. \& Ngwenyama, O. (2014) Advances in Research Methods for Information Systems Research: Data Mining, Data Envelopment Analysis, Value Focused Thinking. Springer Series, New York. 